 \\ TATRA \\ MOUNTaiNS \\ Mathematical Publications
}

\section{ON STABILITY IN GENERALIZED SCHINASI'S MACROECONOMIC MODEL UNDER FIXED EXCHANGE RATES}

\author{
Katarína MakovínYiová - RUdolf ZimkA
}

\begin{abstract}
A nonlinear dynamic macroeconomic model of a small open economy is constructed. The model is based on the IS-LM structure and generalizes the Schinasi's model of a closed economy. Sufficient conditions for the existence of the equilibrium of the model are found and the question of its stability is analyzed.
\end{abstract}

\section{Introduction}

We formulate a nonlinear four dimensional dynamic macroeconomic model of an open economy which describes the development of output, capital, interest rate and money stock. The model has the classical IS-LM structure and expands the three dimensional Schinasi's model of a closed economy [1] to the four dimensional model of an open economy. The model deals with a small economy. It means that economic processes in this economy have negligible influence on those in the region with which it is connected through inter-regional trade and inter-regional capital movement.

The model has the form

$$
\begin{aligned}
\dot{Y} & =\alpha\left[I(Y, K, R)+G-S\left(Y^{D}, R\right)-T(Y)+J(Y)\right], \\
\dot{K} & =I(Y, K, R), \\
\dot{R} & =\beta[L(Y, R)-M], \\
\dot{M} & =J(Y)+\gamma\left(R-R_{f}\right),
\end{aligned}
$$

where $Y$ - output, $K$ - capital stock, $R$ - interest rate of domestic country, $M$ - money supply, $I$ - investments, $G$ - constant government expenditures,

2000 Mathematics Subject Classification: $34 \mathrm{Cxx}, 34 \mathrm{Dxx}, 37 \mathrm{~N} 40$.

Keywords: macroeconomic model, equilibrium, stability.

Supported by the Slovak Grant Agency VEGA No. 1/4633/07. 


\section{KATARIINA MAKOVÍNYIOVÁ - RUDOLF ZIMKA}

$S$ - savings, $T$ - tax collections, $J$ - net export, $L$ - money demand, $R_{f}$ - constant interest rate of a foreign country, $\alpha, \beta, \gamma$ - positive parameters, $t$ - time and

$$
Y^{D}=Y-T(Y), \quad \dot{Y}=\frac{d Y}{d t}, \quad \dot{K}=\frac{d K}{d t}, \quad \dot{R}=\frac{d R}{d t}, \quad \dot{M}=\frac{d M}{d t} .
$$

The economic properties of the functions in (1) are expressed by the following partial derivatives

$$
\begin{gathered}
\frac{\partial I(Y, K, R)}{\partial Y}>0, \quad \frac{\partial I(Y, K, R)}{\partial K}<0, \quad \frac{\partial I(Y, K, R)}{\partial R}<0, \quad \frac{\partial S\left(Y^{D}, R\right)}{\partial Y^{D}}>0 \\
\frac{\partial S\left(Y^{D}, R\right)}{\partial R}>0, \quad \frac{\partial T(Y)}{\partial Y}>0, \quad \frac{\partial J(Y)}{\partial Y}<0 \\
\frac{\partial L(Y, R)}{\partial Y}>0, \quad \frac{\partial L(Y, R)}{\partial R}<0 .
\end{gathered}
$$

It follows from economic theory that parameter $\alpha$ can take small values as output $Y$ reacts rather slow to the changes of the functions involved in the model while parameters $\beta$ and $\gamma$ can take large values as interest rate and capital movement react to the changes of the functions very quickly. This knowledge about possible values of parameters $\alpha, \beta$ and $\gamma$ will be used in the analysis of the stability of the equilibrium. We consider the case of fixed exchange rates. Therefore, the model can be applicable, for example, to the study of economic processes between arbitrary two countries in Eurozone, one of them being much smaller then the second one. The question of the existence of an equilibrium of the model (11) and its stability is solved in the paper.

We assume the following form of functions in the model (1):

$$
\begin{aligned}
I(Y, K, R) & =f_{1}(Y)-i_{2} K-i_{3} R, \\
S\left(Y^{D}, R\right) & =f_{2}\left(Y^{D}\right)+s_{3} R, \\
T(Y) & =t_{1} Y-t_{0}, \\
L(Y, R) & =f_{3}(Y)-l_{3} R,
\end{aligned}
$$

where $f_{1}(Y), f_{2}\left(Y^{D}\right), f_{3}(Y)$, and also $J(Y)$ are nonlinear functions from $C^{2}$, $\frac{d f_{1}(Y)}{d Y}>0, \frac{d f_{2}\left(Y^{D}\right)}{d Y^{D}}>0, \frac{d f_{3}(Y)}{d Y}>0 ; i_{2}, i_{3}, s_{3}, t_{0}, t_{1}, l_{3}$ are positive constants, $0<t_{1}<1$. After substituting (3) into the model (11) we get the model

$$
\begin{aligned}
\dot{Y} & =\alpha\left[f_{1}(Y)-i_{2} K-i_{3} R+G-f_{2}\left(Y^{D}\right)-s_{3} R-t_{1} Y+t_{0}+J(Y)\right] \\
\dot{K} & =f_{1}(Y)-i_{2} K-i_{3} R \\
\dot{R} & =\beta\left[f_{3}(Y)-l_{3} R-M\right] \\
\dot{M} & =J(Y)+\gamma\left(R-R_{f}\right) .
\end{aligned}
$$


ON STABILITY IN GENERALIZED SCHINASI'S MACROECONOMIC MODEL

\section{Existence and stability of an equilibrium}

Consider equations

$$
\begin{aligned}
G-f_{2}(Y)^{D}-s_{3} R-t_{1} Y+t_{0}+J(Y) & =0 \\
J(Y)+\gamma\left(R-R_{f}\right) & =0 .
\end{aligned}
$$

Denote

$$
F(Y, \gamma) \equiv J(Y)\left(\frac{s_{3}}{\gamma}+1\right)-f_{2}\left(Y^{D}\right)-t_{1} Y-s_{3} R_{f}+G+t_{0}
$$

Lemma 1. Let $F(0, \gamma)>0$. Then for sufficiently large $\gamma>0$ there exists a unique solution $\left(Y^{*}(\gamma)>0, R^{*}(\gamma)>0\right)$ of the equations (5).

Pr o of. From the second equation in (5) we have

$$
R=R_{f}-\frac{J(Y)}{\gamma}
$$

Substituting (66) into the first equation in (15) we get $F(Y, \gamma)=0$. This equation has a unique positive solution $Y^{*}(\gamma)$, as

$$
\begin{gathered}
\frac{d F(Y, \gamma)}{d Y}=\left(\frac{s_{3}}{\gamma}+1\right) \frac{d J(Y)}{d Y}-\frac{d f_{2}\left(Y^{D}\right)}{d Y^{D}}\left(1-t_{1}\right)-t_{1}<0 \\
\lim _{Y \rightarrow \infty} F(Y, \gamma)=-\infty \text { and } F(0, \gamma)>0
\end{gathered}
$$

For sufficiently large $\gamma>0$ the value $R^{*}=R^{*}(\gamma)=R_{f}-\frac{J\left(Y^{*}\right)}{\gamma}$ is positive.

LeMma 2. Let the conditions in Lemma 1 be satisfied and $Y^{*}, R^{*}$ be the solutions of the equations (5). If $f_{1}\left(Y^{*}\right)-i_{3} R^{*}>0$ and $f_{3}\left(Y^{*}\right)-l_{3} R^{*}>0$, then the equations

$$
\begin{gathered}
f_{1}\left(Y^{*}\right)-i_{2} K-i_{3} R^{*}=0, \\
f_{3}\left(Y^{*}\right)-l_{3} R^{*}-M=0
\end{gathered}
$$

have a unique positive solutions $K^{*}(\gamma)$ and $M^{*}(\gamma)$.

The assertion of Lemma 2 is clear and it is not necessary to justify it.

On the base of Lemma 1 and Lemma 2 we can formulate the following theorem.

Theorem 1. Let the conditions in Lemma 1 and Lemma 2 be satisfied. Then there exists a unique equilibrium $E^{*}=\left(Y^{*}, K^{*}, R^{*}, M^{*}\right), Y^{*}=Y^{*}(\gamma)>0$, $K^{*}=K^{*}(\gamma)>0, R^{*}=R^{*}(\gamma)>0, M^{*}=M^{*}(\gamma)>0$ of the model (44). 


\section{KATARÍNA MAKOVÍNYIOVÁ - RUDOLF ZIMKA}

Suppose the model (4) has the unique positive equilibrium $E^{*}(\gamma)=\left(Y^{*}(\gamma)\right.$, $\left.K^{*}(\gamma), R^{*}(\gamma), M^{*}(\gamma)\right), Y^{*}(\gamma)>0, K^{*}(\gamma)>0, R^{*}(\gamma)>0, M^{*}(\gamma)>0$. Let us transform this equilibrium $E^{*}(\gamma)$ into the origin $E_{1}^{*}=\left(Y^{*}=0, K^{*}=0\right.$, $R^{*}=0, M^{*}=0$ ) by shifting

$$
Y_{1}=Y-Y^{*}, \quad K_{1}=K-K^{*}, \quad R_{1}=R-R^{*}, \quad M_{1}=M-M^{*} .
$$

Then the model (4) obtains the following form

$$
\begin{aligned}
\dot{Y}_{1}= & \alpha\left[f_{1}\left(Y_{1}+Y^{*}\right)-f_{2}\left(\left(Y_{1}+Y^{*}\right)^{D}\right)+J\left(Y_{1}+Y^{*}\right)\right] \\
& +\alpha\left[-t_{1} Y_{1}-i_{2} K_{1}-\left(i_{3}+s_{3}\right) R_{1}\right] \\
& +\alpha\left[-t_{1} Y^{*}-i_{2} K^{*}-\left(i_{3}+s_{3}\right) R^{*}+t_{0}+G\right], \\
\dot{K}_{1}= & f_{1}\left(Y_{1}+Y^{*}\right)-i_{2} K_{1}-i_{3} R_{1}-i_{2} K^{*}-i_{3} R^{*}, \\
\dot{R}_{1}= & \beta\left[f_{3}\left(Y_{1}+Y^{*}\right)-l_{3} R_{1}-M_{1}-l_{3} R^{*}-M^{*}\right], \\
\dot{M}_{1}= & J\left(Y_{1}+Y^{*}\right)+\gamma\left(R_{1}+R^{*}-R_{f}\right) .
\end{aligned}
$$

The Jacobian matrix $A=A(\alpha, \beta, \gamma)$ of the model (7) at the equilibrium $E_{1}^{*}$ is

$$
\mathbf{A}(\alpha, \beta, \gamma)=\left(\begin{array}{cccc}
\alpha\left(f_{1 Y}-f_{2 Y}+J_{Y}-t_{1}\right) & -\alpha i_{2} & -\alpha\left(i_{3}+s_{3}\right) & 0 \\
f_{1 Y} & -i_{2} & -i_{3} & 0 \\
\beta f_{3 Y} & 0 & -\beta l_{3} & -\beta \\
J_{Y} & 0 & \gamma & 0
\end{array}\right),
$$

where

$$
f_{1 Y}=\frac{d f_{1}\left(E^{*}\right)}{d Y}, \quad f_{2 Y}=\frac{d f_{2}\left(E^{* D}\right)}{d Y^{D}}\left(1-t_{1}\right), \quad f_{3 Y}=\frac{d f_{3}\left(E^{*}\right)}{d Y}, \quad J_{Y}=\frac{d J\left(E^{*}\right)}{d Y} .
$$

The characteristic equation of $\mathbf{A}(\alpha, \beta, \gamma)$ is given by

$$
\lambda^{4}+a_{1}(\alpha, \beta, \gamma) \lambda^{3}+a_{2}(\alpha, \beta, \gamma) \lambda^{2}+a_{3}(\alpha, \beta, \gamma) \lambda+a_{4}(\alpha, \beta, \gamma)=0,
$$

where

$$
\begin{aligned}
a_{1}= & \alpha\left(t_{1}+f_{2 Y}-J_{Y}-f_{1 Y}\right)+\beta l_{3}+i_{2}, \\
a_{2}= & \alpha \beta\left(l_{3}\left(t_{1}+f_{2 Y}-J_{Y}-f_{1 Y}\right)+f_{3 Y}\left(i_{3}+s_{3}\right)\right)+\alpha i_{2}\left(t_{1}+f_{2 Y}-J_{Y}\right) \\
& +\beta\left(\gamma+i_{2} l_{3}\right) \\
a_{3}= & \beta\left(\alpha \gamma\left(t_{1}+f_{2 Y}-J_{Y}-f_{1 Y}\right)\right. \\
& \left.+\alpha\left(i_{2} l_{3}\left(t_{1}+f_{2 Y}-J_{Y}\right)+i_{2} s_{3} f_{3 Y}-J_{Y}\left(i_{3}+s_{3}\right)\right)+\gamma i_{2}\right), \\
a_{4}= & \alpha \beta \gamma i_{2}\left(t_{1}+f_{2 Y}-J_{Y}\right)-\alpha \beta i_{2} s_{3} J_{Y} .
\end{aligned}
$$

The Routh-Hurwitz conditions which are necessary and sufficient for all the roots of (8) to have negative real parts, are equivalent to the conditions

$$
a_{1}>0, \quad a_{2}>0, \quad a_{4}>0,
$$




\section{ON STABILITY IN GENERALIZED SCHINASI'S MACROECONOMIC MODEL}

$$
\Delta_{3}=\left(a_{1} a_{2}-a_{3}\right) a_{3}-a_{1}^{2} a_{4}>0 .
$$

The conditions (9) are satisfied for an arbitrary $\alpha>0$ and sufficiently large positive $\beta, \gamma$.

As parameter $\beta$ can be very large, let us order $\Delta_{3}$ as a polynomial with respect to $\beta$. Denoting

$$
\begin{aligned}
& a_{1}=a_{11} \beta+a_{12}(\alpha), \\
& a_{2}=a_{21}(\alpha, \gamma) \beta+a_{22}(\alpha), \\
& a_{3}=a_{31}(\alpha, \gamma) \beta, \\
& a_{4}=a_{41}(\alpha, \gamma) \beta,
\end{aligned}
$$

the relation $\Delta_{3}$ can be expressed in the form

$$
\Delta_{3}(\alpha, \beta, \gamma)=g_{1}(\alpha, \gamma) \beta^{2}+g_{2}(\alpha, \gamma) \beta+g_{3}(\alpha, \gamma)
$$

where

$$
\begin{aligned}
& g_{1}(\alpha, \gamma)=\left(a_{21} a_{31}-a_{11} a_{41}\right) a_{11} \\
& g_{2}(\alpha, \gamma)=\left(\left(a_{12} a_{21}+a_{11} a_{22}\right) a_{31}-2 a_{11} a_{12} a_{41}-a_{31}^{2}\right) \\
& g_{3}(\alpha, \gamma)=a_{12} a_{22} a_{31}-a_{12}^{2} a_{41} .
\end{aligned}
$$

Express the coefficient

$$
g_{1}(\alpha, \gamma)=\left(a_{21} a_{31}-a_{11} a_{41}\right) a_{11}
$$

in the form

$$
g_{1}(\alpha, \gamma)=\mathcal{A} \alpha^{2}+\mathcal{B} \alpha+\mathcal{C}
$$

where

$$
\begin{aligned}
& \mathcal{A}=\mathcal{A}(\gamma)=\left(l_{3} A+\left(i_{3}+s_{3}\right) f_{3 Y}\right)\left(\gamma A+i_{2} l_{3} B+i_{2} s_{3} f_{3 Y}-\left(i_{3}+s_{3}\right) J_{Y}\right) l_{3}, \\
& \mathcal{B}=\mathcal{B}(\gamma)=\left(\left(\gamma+i_{2} l_{3}\right)\left(\gamma A+i_{2} l_{3} B+i_{2} s_{3} f_{3 Y}-\left(i_{3}+s_{3}\right) J_{Y}\right)\right. \\
& \left.+\gamma i_{2}\left(l_{3} A+\left(i_{3}+s_{3}\right) f_{3 Y}\right)-l_{3}\left(\gamma i_{2} B-i_{2} s_{3} J_{Y}\right)\right) l_{3}, \\
& \mathcal{C}=\mathcal{C}(\gamma)=\gamma i_{2}\left(\gamma+i_{2} l_{3}\right) l_{3} \\
& A=t_{1}+f_{2 Y}-J_{Y}-f_{1 Y} \text {, } \\
& B=t_{1}+f_{2 Y}-J_{Y} \text {. }
\end{aligned}
$$

The expression $A$ can be positive, zero or negative. If $A \geq 0$, then we can formulate the following theorem.

Theorem 2. Let $A$ be nonnegative. Take parameters $\alpha$ and $\gamma$ at any positive level. Then the equilibrium $E^{*}$ is asymptotically stable for sufficiently large positive $\beta$. 


\section{KATARÍNA MAKOVÍNYIOVÁ - RUDOLF ZIMKA}

P r o of. If $A \geq 0$, then the expression $g_{1}(\alpha, \gamma)=\left(a_{21} a_{31}-a_{11} a_{41}\right) a_{11}$ is positive. As

$$
\frac{\Delta_{3}(\alpha, \beta, \gamma)}{\beta^{2}}=\left(a_{21} a_{31}-a_{11} a_{41}\right) a_{11}+h(\alpha, \beta, \gamma),
$$

where $\lim _{\beta \rightarrow \infty} h(\alpha, \beta, \gamma)=0$ for arbitrary $\alpha$ and $\gamma, \Delta_{3}(\alpha, \beta, \gamma)>0$ for sufficiently large $\beta$.

As $\mathrm{K}$ a ld or showed [2, in practice the value $f_{1 Y}$ is considerably bigger than the value $f_{2 Y}$ at the equilibrium $E^{*}$, what causes that the value $A$ is quite often negative. Suppose now that $A<0$. In this case the stability or unstability of the equilibrium $E^{*}$ depends on the values of $\alpha, \beta$ and $\gamma$. To analyze this situation rigorously we shall utilize the Liu's necessary and sufficient conditions for the Jacobian matrix $\mathbf{A}(\alpha, \beta, \gamma)$ to have a pair of purely imaginary eigenvalues and the rest two with negative real parts [3], which are equivalent to the conditions

$$
\begin{gathered}
a_{1}>0, \quad a_{2}>0, \quad a_{4}>0, \\
\Delta_{3}=\left(a_{1} a_{2}-a_{3}\right) a_{3}-a_{1}^{2} a_{4}=0 .
\end{gathered}
$$

As it was mentioned before, the conditions (12) are satisfied for an arbitrary $\alpha>0$ and sufficiently large positive $\beta$ and $\gamma$. Let us analyze first the equation

$$
g_{1}(\alpha, \gamma)=\mathcal{A} \alpha^{2}+\mathcal{B} \alpha+\mathcal{C}=0
$$

The coefficient $\mathcal{C}$ is always positive, the coefficient $\mathcal{B}$ is negative for sufficiently large $\gamma$, and the sign of $\mathcal{A}$ depends on the sign of $l_{3} A+\left(i_{3}+s_{3}\right) f_{3 Y}$ for sufficiently large $\gamma$. The following 3 cases can happen:

Case 1. $l_{3} A+\left(i_{3}+s_{3}\right) f_{3 Y}>0$.

Then $\mathcal{A}<0$ and the equation (14) has two roots of different sign

$$
\alpha_{1}^{(1)}=\frac{-\mathcal{B}+\sqrt{\mathcal{B}^{2}-4 \mathcal{A C}}}{2 \mathcal{A}}<0, \quad \alpha_{1}^{(2)}=\frac{-\mathcal{B}-\sqrt{\mathcal{B}^{2}-4 \mathcal{A C}}}{2 \mathcal{A}}>0 .
$$

Case 2. $l_{3} A+\left(i_{3}+s_{3}\right) f_{3 Y}=0$.

Then $\mathcal{A}=0$ and the equation (14) has positive root

$$
\alpha_{2}^{(1)}=-\frac{\mathcal{C}}{\mathcal{B}}>0 .
$$

Case 3. $l_{3} A+\left(i_{3}+s_{3}\right) f_{3 Y}<0$.

Then $\mathcal{A}>0$ and $\mathcal{B}^{2}-4 \mathcal{A C}>0$ for sufficiently large $\gamma$, because $\mathcal{B}^{2}-4 \mathcal{A C}=$ $A^{2} \gamma^{4}+g(\gamma), \lim _{\gamma \rightarrow \infty} \frac{g(\gamma)}{\gamma^{4}}=0$. The equation (14) has two positive roots

$$
\alpha_{3}^{(1)}=\frac{-\mathcal{B}-\sqrt{\mathcal{B}^{2}-4 \mathcal{A C}}}{2 \mathcal{A}}>0, \quad \alpha_{3}^{(2)}=\frac{-\mathcal{B}+\sqrt{\mathcal{B}^{2}-4 \mathcal{A C}}}{2 \mathcal{A}}>0 .
$$




\section{ON STABILITY IN GENERALIZED SCHINASI'S MACROECONOMIC MODEL}

Consider now an arbitrary $\gamma$ but such that the conditions (12) are satisfied, $\mathcal{B}<0$ and $\mathcal{B}^{2}-4 \mathcal{A C}>0$, denote it $\gamma^{*}$ and fix it. Take $\delta=\frac{1}{\beta}$ and create the function

$$
F\left(\alpha, \delta, \gamma^{*}\right)=\delta^{2} \Delta_{3}\left(\alpha, \frac{1}{\delta}, \gamma^{*}\right)=g_{1}\left(\alpha, \gamma^{*}\right)+g_{2}\left(\alpha, \gamma^{*}\right) \delta+g_{3}\left(\alpha, \gamma^{*}\right) \delta^{2} .
$$

It holds:

(1) $F\left(\alpha_{i}^{(j)}, 0, \gamma^{*}\right)=g_{1}\left(\alpha_{i}^{(j)}, \gamma^{*}\right)=0, i=1,2,3 ; j=1,2, i \neq j$, where $\alpha_{i}^{(j)}$ are the positive roots of the equation (14).

(2) For $\frac{\partial F\left(\alpha_{i}^{(j)}, 0, \gamma^{*}\right)}{\partial \alpha}$ we get:

Case 1.

$$
\frac{\partial F\left(\alpha_{1}^{(2)}, 0, \gamma^{*}\right)}{\partial \alpha}=2 \alpha_{1}^{(2)} \mathcal{A}+\mathcal{B}=2 \frac{-\mathcal{B}-\sqrt{\mathcal{B}^{2}-4 \mathcal{A C}}}{2 \mathcal{A}} \mathcal{A}+\mathcal{B}=-\sqrt{\mathcal{B}^{2}-4 \mathcal{A C}}<0 .
$$

Case 2.

$$
\frac{\partial F\left(\alpha_{2}^{(1)}, 0, \gamma^{*}\right)}{\partial \alpha}=\mathcal{B}<0 \text {. }
$$

Case 3.

$$
\begin{aligned}
& \frac{\partial F\left(\alpha_{i}^{(j)}, 0, \gamma^{*}\right)}{\partial \alpha}=2 \alpha_{i}^{(j)} \mathcal{A}+\mathcal{B}: \\
& \text { a) } \frac{\partial F\left(\alpha_{3}^{(1)}, 0, \gamma^{*}\right)}{\partial \alpha}=2 \frac{-\mathcal{B}-\sqrt{\mathcal{B}^{2}-4 \mathcal{A C}}}{2 \mathcal{A}} \mathcal{A}+\mathcal{B}=-\sqrt{\mathcal{B}^{2}-4 \mathcal{A C}}<0, \\
& \text { b) } \frac{\partial F\left(\alpha_{3}^{(2)}, 0, \gamma^{*}\right)}{\partial \alpha}=2 \frac{-\mathcal{B}+\sqrt{\mathcal{B}^{2}-4 \mathcal{A C}}}{2 \mathcal{A}} \mathcal{A}+\mathcal{B}=+\sqrt{\mathcal{B}^{2}-4 \mathcal{A C}}>0 .
\end{aligned}
$$

On the base of Implicit Functional Theorem we can formulate the following lemma.

Lemma 3. To an arbitrary sufficiently small positive $\delta^{*}$ there exists in all cases 1-3 $\alpha^{*}$ such that

$$
F\left(\alpha^{*}, \delta^{*}, \gamma^{*}\right)=\delta^{*} \Delta_{3}\left(\alpha^{*}, \frac{1}{\delta^{*}}, \gamma^{*}\right)=0
$$

what means that

$$
\Delta_{3}\left(\alpha^{*}, \frac{1}{\delta^{*}}, \gamma^{*}\right)=\Delta_{3}\left(\alpha^{*}, \beta^{*}, \gamma^{*}\right)=0, \quad \beta^{*}=\frac{1}{\delta^{*}} .
$$

Now let us consider a triple $\left(\alpha^{*}, \beta^{*}, \gamma^{*}\right)$ of parameters $\alpha, \beta, \gamma$ with sufficiently large $\beta^{*}$, such that $\Delta_{3}\left(\alpha^{*}, \beta^{*}, \gamma^{*}\right)=0$, existence is guaranteed by Lemma 3 . Fix $\beta^{*}$ and $\gamma^{*}$ and investigate the question of stability of $E^{*}$ on the interval $\left(\alpha^{*}-\varepsilon, \alpha^{*}+\varepsilon\right), \varepsilon>0$.

Case 1.

Because $\frac{\partial F\left(\alpha_{1}^{(2)}, 0, \gamma^{*}\right)}{\partial \alpha}<0$, it follows from the continuity of the function $\frac{\partial F(\alpha, \delta, \gamma)}{\partial \alpha}$ that for $\delta^{*}>0$, which is sufficiently close to $\delta=0$, also $\frac{\partial F\left(\alpha^{*}, \delta^{*}, \gamma^{*}\right)}{\partial \alpha}=$ $\delta^{*} \frac{\partial \Delta_{3}\left(\alpha^{*}, \frac{1}{\left.\delta^{*}, \gamma^{*}\right)}\right.}{\partial \alpha}<0$, what means that $\frac{\partial \Delta_{3}\left(\alpha^{*}, \beta^{*}, \gamma^{*}\right)}{\partial \alpha}<0$. 


\section{KATARÍNA MAKOVÍNYIOVÁ - RUDOLF ZIMKA}

Analogously, taking into account cases $2-3$, we get that for $\delta^{*}$ sufficiently close to $\delta=0$ there is:

Case 2.

$\frac{\partial \Delta_{3}\left(\alpha^{*}, \beta^{*}, \gamma^{*}\right)}{\partial \alpha}<0$.

Case 3.
a) $\frac{\partial \Delta_{3}\left(\alpha^{*}, \beta^{*}, \gamma^{*}\right)}{\partial \alpha}<0$,
b) $\frac{\partial \Delta_{3}\left(\alpha^{*}, \beta^{*}, \gamma^{*}\right)}{\partial \alpha}>0$.

Performed considerations enable us to state the next theorem.

TheOREM 3. The equilibrium $E^{*}$ of the model (4) in a small neighborhood of the critical value $\alpha^{*}$ is the following:

Case 1. asymptotically stable for $\alpha<\alpha^{*}$ and unstable for $\alpha>\alpha^{*}$.

Case 2. asymptotically stable for $\alpha<\alpha^{*}$ and unstable for $\alpha>\alpha^{*}$.

Case 3.

a) asymptotically stable for $\alpha<\alpha^{*}$ and unstable for $\alpha>\alpha^{*}$,

b) unstable for $\alpha<\alpha^{*}$ and asymptotically stable for $\alpha>\alpha^{*}$.

\section{REFERENCES}

[1] SCHINASI, G. J.: Fluctuations in a dynamic, intermediate-run IS-LM model: applications of the Poincaré-Bendixon theorem, J. Econom. Theory 28 (1982), 369-375.

[2] KALDOR, N.: A model of the trade cycle, Economic J. 50 (1940), 69-86.

[3] LIU, W. M.: Criterion of Hopf bifurcations without using eigenvalues, J. Math. Anal. Appl. 182 (1994), 250-256.

Received September 30, 2008

\author{
Katarína Makovínyiová \\ Department of Mathematics and \\ Descriptive Geometry \\ Technical University in Zvolen \\ T. G. Masaryka 2117/24 \\ SK-960-53 Zvolen \\ SLOVAKIA \\ E-mail: kmakovin@vsld.tuzvo.sk \\ Rudolf Zimka \\ Department of Quantitative Methods and \\ Informatics \\ Faculty of Economics \\ Matej Bel University \\ Tajovského 10 \\ SK-975-90 Banská Bystrica \\ SLOVAKIA \\ E-mail: rudolf.zimka@umb.sk
}

